\title{
Use of dabigatran vs. warfarin with low-molecular-weight heparin bridging in catheter ablation for atrial fibrillation patients with a low CHADS2 score
}

\author{
LI HAO ${ }^{1,2}$, BING RONG ${ }^{1,2}$, FEI XIE ${ }^{1,2}$, MING-JIE LIN ${ }^{1,2}$ and JING-QUAN ZHONG ${ }^{1,2}$ \\ ${ }^{1}$ The Key Laboratory of Cardiovascular Remodeling and Function Research, \\ Chinese Ministry of Education and Chinese Ministry of Health, the State and Shandong Province Joint \\ Key Laboratory of Translational Cardiovascular Medicine, Qilu Hospital of Shandong University; \\ ${ }^{2}$ Department of Cardiology, Qilu Hospital, Shandong University, Jinan, Shandong 250012, P.R. China
}

Received November 3, 2016; Accepted February 21, 2017

DOI: $10.3892 /$ br.2017.880

\begin{abstract}
The purpose of the present study was to compare the efficacy and safety of dabigatran and interrupted warfarin with low-molecular-weight heparin bridging in non-valvular atrial fibrillation (AF) catheter ablation. Previously, there has been concerns that bridging therapy increases bleeding events without the benefit of stroke prevention. It has been suggested that bridging therapy should be considered only for patients at high-risk of thrombosis. Nevertheless, bridging therapy in AF patients with a low CHADS2 score may be safe and effective. The authors performed a prospective, observational study that included consecutive 240 patients undergoing AF ablation in P.R. China. A total of 139 patients received $110 \mathrm{mg}$ dabigatran twice daily and 101 patients took dose-adjusted warfarin that had been bridged with low-molecular-weight heparin. The mean patient age was 55.48 years with $72.1 \%$ being men and $74.2 \%$ having paroxysmal AF. One thromboembolic complication occurred in the dabigatran group compared to none in the warfarin group. Both the groups presented a similar major bleeding rate, total bleeding rate, and bleeding and thromboembolic complications. In patients undergoing AF ablation, the risk of bleeding or thromboembolic complications was similar for both dabigatran and interrupted warfarin with bridging therapy. Bridging therapy appeared to be safe and effective for the low-risk population.
\end{abstract}

Correspondence to: Dr Jing-Quan Zhong, Department of Cardiology, Qilu Hospital, Shandong University, 107 West Culture Road, Jinan, Shandong 250012, P.R. China

E-mail: 18560086597@163.com

Key words: non-valvular atrial fibrillation, catheter ablation, bridging therapy, dabigatran, warfarin, low CHADS2 score

\section{Introduction}

Atrial fibrillation (AF) is the most commonly encountered cardiac arrhythmia in clinical practice and is associated with significantly increased morbidity and mortality $(1,2)$. Catheter-based AF ablation with the primary aim of pulmonary vein (PV) electrical isolation has been established as a treatment option for patients with symptomatic, drug-refractory $\mathrm{AF}(3,4)$. AF ablation is a relatively complex procedure, and is associated with the potential risk of periprocedural thromboembolic complications. The endothelial lesion caused by the ablation energy may serve a significant role in activating the clotting cascade. In addition, the cardioversion or the restored contractility post-ablation may dislodge left atrial microthrombi $(5,6)$. However, minimizing thromboembolic complications with periprocedural anticoagulation could potentially increase the risk of bleeding events. As such, maintaining the balance between bleeding and thrombosis is critical to the safety of the ablation procedure.

Warfarin administration has been the mainstay for AF ablation anticoagulation, with a target international normalized ratio (INR) level of 2.0 to 3.0. Bridging anticoagulation refers to the temporary interruption of oral anticoagulation and introduction of a short-acting anticoagulant such as low-molecular-weight heparin (LMWH). Recently, uninterrupted therapeutic warfarin has been demonstrated to be associated with less bleeding events, and it has the benefit of preventing stroke in patients undergoing catheter ablation of AF compared to use of warfarin with bridging LMWH (7). In patients with AF who need to cease warfarin treatment in preparation for an invasive procedure, forgoing bridging therapy was non-inferior to perioperative bridging with LMWH for reducing the risk of stroke and major bleeding (8). The current international guidelines suggest that bridging therapy should be considered for patients at high risk of thrombosis; low-risk patients do not require bridging (9-11). The CHADS2 score can be calculated to assess the risk of stroke. Low-risk patients include those with a CHADS2 score of 0-2, moderate-risk with a CHADS2 score of 3-4, and high-risk with a CHADS2 score of 5 or 6 . Previous 
studies have demonstrated that Asian patients are more sensitive to the anticoagulant effects of warfarin (12) and warfarin has a long half-life, uninterrupted warfarin without bridging therapy remains controversial and has not been widely accepted in China. Conversely, the direct thrombin inhibitor dabigatran has more predictable pharmacokinetics and the unique property of a rapid onset and short half-life. Therefore, it may not necessitate bridging therapy, and uninterrupted administration appears to be easier to achieve. Although the availability of these new oral anticoagulants (OACs) would obviate the need for bridging, the use of new OACs is limited due to their high medical cost.

\section{Materials and methods}

Inclusion and exclusion criteria. A prospective, observational study was performed using patients undergoing AF ablation at an electrophysiology center (at Qilu Hospital, Shandong University, Jinan, Shandong) in China between July 2013 and June 2015. The research protocol was approved by the institutional review board of Qilu Hospital (Jinan, China) and the signed informed consent was provided by each patient. The inclusion criteria were: i) Age 18-75 years, ii) presence of AF as evidenced by a 12-lead electrocardiogram or $24 \mathrm{~h}$ Holter monitoring, iii) absence of thrombus in atrium/atrial appendage by transesophageal echocardiography (TEE) and iv) hemodynamic stable non-valvular atrial fibrillation. The exclusion criteria were: i) Hypersensitivity to the active ingredient or any excipients, ii) patients with severe renal impairment (creatinine clearance $<30 \mathrm{ml} / \mathrm{min}$ ), iii) clinically active bleeding, iv) significant risk factors for major bleeding, v) concomitant treatment with any other anticoagulants except switching therapy to or from dabigatran, or when unfractionated heparin is given to maintain an open central venous or arterial catheter, vi) severe hepatic impairment or liver disease, vii) concomitant treatment with systemic ketoconazole, cyclosporine, itraconazole, tacrolimus and dronedarone, and viii) a prosthetic heart valve or hemodynamically significant valvular disease. The study included 240 patients with drug-refractory, paroxysmal or persistent AF who underwent AF catheter ablation. Among them, 137 patients received $110 \mathrm{mg}$ dabigatran twice a day (group D) and the remaining 103 received dose-adjusted warfarin (group W). The design flow chart is presented in Fig. 1.

Periprocedural anticoagulation. In group D, dabigatran was not discontinued until the morning of the procedure; it was restarted $4 \mathrm{~h}$ following hemostasis at the same dosage as was given previously that morning. In group $\mathrm{W}$, all patients received dose-adjusted warfarin with a target INR of 2.0-3.0. Warfarin was discontinued 3 days prior to the procedure and patients were bridged with subcutaneous LMWH until the evening before the ablation procedure. The administration of LMWH was restarted on the evening of the procedure and continued until an INR of 2.0-3.0 was achieved. Warfarin treatment was restarted on the evening of or the day after the procedure. For all patients in the two groups, TEE was performed prior to the procedure to exclude the presence of an intracardiac thrombus.
Ablation procedure. Before the transseptal puncture, intraprocedural anticoagulation was conducted with a weight-based (80-100 U/kg) heparin bolus administered intravenously. Based on the activated clotting time (ACT), infusion of heparin continuously at 1,000 U/h was given and adjusted, which was monitored every $30 \mathrm{~min}$ and targeted at 300 to $350 \mathrm{sec}$. Meanwhile, the transseptal sheaths were continuously infused with heparinized saline. Vascular access was achieved through the right femoral vein and left subclavian vein. A circular mapping catheter was performed to guide pulmonary vein antrum isolation. Electrical activity recorded by placing a circular mapping catheter (Lasso, Biosense Webster, Inc. Diamond Bar, CA, USA) in the ostium of each pulmonary vein $(\mathrm{PV})$, and pulmonary venography was performed for each PV. Following successful isolation of all the PVs, burst atrial pacing was performed to confirm that an atrial arrhythmia was induced. Additional procedures including the complex fractionated atrial electrograms ablation, linear ablation of the LA, and the superior vena cava isolation were performed, if AF/atrial tachyarrhythmia was induced and sustained. Either the elimination or dissociation of the PV potentials was the end-point of the PV isolation, recorded through the circular catheters placed within the PVs. If termination was unsuccessful, cardioversion was performed to restore the sinus rhythm. During the procedure, the blood pressure was noninvasively monitored.

Follow-up. Following the ablation, the patients leave hospital in 3 to 5 days, and were checked at the outpatient clinic 1 week and 1 month after the ablation for any postprocedural complications. Thromboembolic complications defined as ischemic stroke, transient ischemic attack (TIA), or systemic thromboembolism were the primary end-points (efficacy end-points). Major (requiring a transfusion or surgical intervention) and minor bleeding complications were the secondary end-points (safety end-points).

Statistical analysis. Statistical analyses were performed using SPSS software (version, 17.0; SPSS Inc., Chicago, IL, USA). Continuous variables are presented as the as mean \pm standard deviation and compared using a Student's t-test or Mann-Whitney U test. Categorical variables were presented as counts and percentages then analyzed using either a Chi-squared test or Fisher's exact test. Bleeding complications (minor and major), thromboembolic complications and total complications were compared between groups. $\mathrm{P}<0.05$ (two-sided) was considered to indicate a statistically significant difference.

\section{Results}

Patient characteristics. A total of 240 patients presenting with AF between July 2013 and June 2015 were enrolled in the present study. Patients were treated according to their preference to undergo the procedure with an anticoagulation strategy of dabigatran (group $\mathrm{D}, \mathrm{n}=139$ ) or warfarin with bridging therapy (group $\mathrm{W}, \mathrm{n}=101$ ). The baseline characteristics of the two groups were similar with no significant differences in age, sex, body mass index, blood pressure, left atrial size, left ventricular ejection fraction, CHADS2 
Table I. Patient baseline characteristics.

\begin{tabular}{|c|c|c|c|}
\hline & $\begin{array}{l}\text { Dabigatran group } \\
\qquad(\mathrm{n}=139)\end{array}$ & $\begin{array}{l}\text { Warfarin group } \\
\qquad(n=101)\end{array}$ & P-value \\
\hline Age (years) & $55.12 \pm 11.10$ & $55.96 \pm 10.40$ & 0.554 \\
\hline Male $(\%)$ & $101(73)$ & $72(71)$ & 0.815 \\
\hline Body mass index $\left(\mathrm{kg} / \mathrm{m}^{2}\right)$ & $27.25 \pm 3.78$ & $26.32 \pm 3.49$ & 0.055 \\
\hline \multicolumn{4}{|l|}{ Blood pressure (mmHg) } \\
\hline Systolic & $132.87 \pm 18.33$ & $130.70 \pm 17.55$ & 0.358 \\
\hline Diastolic & $82.14 \pm 13.37$ & $80.47 \pm 15.21$ & 0.368 \\
\hline Persistent atrial fibrillation (\%) & $33(24)$ & $29(29)$ & 0.385 \\
\hline Duration of atrial fibrillation (months) & $37.08 \pm 51.69$ & $45.34 \pm 41.74$ & 0.037 \\
\hline Hypertension (\%) & $64(46)$ & $43(43)$ & 0.594 \\
\hline Diabetes $(\%)$ & $18(13)$ & $16(16)$ & 0.526 \\
\hline Coronary artery disease $(\%)$ & $9(7)$ & $13(13)$ & 0.090 \\
\hline Heart failure $(\%)$ & $2(1)$ & $1(1)$ & $P>0.99$ \\
\hline Transient ischemic attacks or stroke (\%) & $6(4)$ & $8(8)$ & 0.240 \\
\hline Smoking $(\%)$ & $60(43)$ & $30(30)$ & 0.033 \\
\hline Alcohol drinking (\%) & $37(27)$ & $24(24)$ & 0.616 \\
\hline Alanine aminotransferase (U/l) & $22.05 \pm 11.87$ & $22.36 \pm 11.12$ & 0.840 \\
\hline Serum creatinine $(\mu \mathrm{mol} / \mathrm{l})$ & $71.63 \pm 14.06$ & $69.74 \pm 11.59$ & 0.272 \\
\hline Fasting blood glucose (mmol/1) & $5.03 \pm 1.00$ & $5.26 \pm 1.74$ & 0.202 \\
\hline Platelet count $\left(\times 10^{9} / 1\right)$ & $218.24 \pm 45.20$ & $208.76 \pm 47.59$ & 0.118 \\
\hline INR & $1.04 \pm 0.13$ & $1.07 \pm 0.23$ & 0.900 \\
\hline APTT-S & $31.22 \pm 8.80$ & $32.21 \pm 4.23$ & 0.649 \\
\hline CHADS2 score ${ }^{a}$ & $0.68 \pm 0.80$ & $0.77 \pm 0.89$ & 0.598 \\
\hline CHADS2 $\geq 2(n, \%)$ & $17(12)$ & $19(19)$ & 0.159 \\
\hline CHA2DS2VASc score ${ }^{b}$ & $1.14 \pm 1.17$ & $1.32 \pm 1.21$ & 0.241 \\
\hline CHA2DS2VASc $\geq 2(n, \%)$ & $42(30)$ & $35(35)$ & 0.467 \\
\hline HAS-BLED score ${ }^{c}$ & $1.04 \pm 0.96$ & $1.11 \pm 1.10$ & 0.900 \\
\hline HAS-BLED $\geq 3(\mathrm{n}, \%)$ & $8(6)$ & $15(15)$ & 0.018 \\
\hline Left atrial size $(\mathrm{mm})$ & $39.75 \pm 5.83$ & $39.51 \pm 5.63$ & 0.746 \\
\hline Left ventricular ejection fraction (\%) & $61.49 \pm 5.03$ & $60.56 \pm 6.28$ & 0.207 \\
\hline \multicolumn{4}{|l|}{ AF ablation procedure } \\
\hline Cryoablation & $28(20)$ & $15(15)$ & 0.291 \\
\hline Intraprocedural cardioversion & $22(16)$ & $18(18)$ & 0.682 \\
\hline Anticoagulation-experienced (n, \%) & $19(14)$ & $13(13)$ & 0.858 \\
\hline Warfarin $(\mathrm{n}, \%)$ & $3(2)$ & $9(9)$ & 0.038 \\
\hline Dabigatran (n, \%) & $16(12)$ & $4(4)$ & 0.242 \\
\hline
\end{tabular}

${ }^{a}$ CHADS2 score [congestive heart failure; hypertension; age $\geq 75$ years; type 2 diabetes; and previous stroke, transient ischemic attack, or thromboembolism (doubled)]. ${ }^{\mathrm{b}} \mathrm{CHA} 2 \mathrm{DS} 2-\mathrm{VASc}$ score [congestive heart failure; hypertension; age $\geq 75$ years (doubled); type 2 diabetes; and previous stroke, transient ischemic attack, or thromboembolism (doubled); vascular disease; age 65-75 years; and sex category (female)]. ${ }^{\mathrm{c}} \mathrm{HAS}-\mathrm{BLED}$ score (hypertension, abnormal renal/liver function, stroke, bleeding history or predisposition, labile international normalized ratio, elderly, drugs/alcohol concomitantly). AF, trial fibrillation.

score, CHA2DS2VASc score, alcohol consumption, proportion of patients with persistent AF or the number of patients with a history of previous stroke/TIA (Table I). In group D, 8 patients $(6 \%)$ had a HAS-BLED $\geq 3$ compared with $15(15 \%)$ in group $\mathrm{W}(\mathrm{P}<0.05)$. Those receiving dabigatran were more likely to be smokers and had a shorter duration of atrial fibrillation, when compared with the warfarin patients. During the procedure, 28 patients $(20 \%)$ in group D and 15 patients $(15 \%)$ in group $\mathrm{W}(\mathrm{P}>0.05)$ received cryoablation. Intraprocedural cardioversions were conducted in 22 patients receiving dabigatran (16\%), compared with 18 patients receiving warfarin $(18 \%, \mathrm{P}>0.05)$.

Study outcomes. Complications associated with the procedures are presented in Table II. A periprocedural stroke event occurred in one patient in the dabigatran group and 
Table II. Comparison of complications between patients on dabigatran and warfarin.

\begin{tabular}{lcccr}
\hline & Dabigatran (n=139) & Warfarin (n=101) & Total (n=240) & P-value \\
\hline Major bleeding complications (n, \%) & 0 & 0 & 0 & \\
Cardiac tamponade & 0 & 0 & 0 & \\
Intracranial bleeding & 0 & 0 & 0 & \\
Extracranial & 0 & 0 & 0 & 0.215 \\
Minor bleeding complications (n, \%) & $23(17)$ & $11(11)$ & $34(14)$ & 0.364 \\
Groin hematoma & $11(8)$ & $5(5)$ & $16(7)$ & $>0.050$ \\
Hemothorax & $1(1)$ & $1(1)$ & $2(1)$ & $>0.050$ \\
Hemoptysis & $1(1)$ & 0 & $1(1)$ & 0.643 \\
Urogenital bleeding & $7(5)$ & $3(3)$ & $5(4)$ & $>0.050$ \\
Gastrointestinal bleeding & $3(2)$ & $2(2)$ & $34(14)$ & 0.215 \\
Total bleeding complications (n, \%) & $23(17)$ & $11(11)$ & $1(1)$ & $>0.050$ \\
Embolic complications (stroke/TIA) (n, \%) & $1(1)$ & 0 & $35(15)$ & 0.167 \\
Composite of bleeding and embolic complications & $24(17)$ & $11(11)$ & $5(2)$ & 0.076 \\
Other complications (n, \%) & $5(4)^{\mathrm{a}}$ & 0 & \\
\hline
\end{tabular}

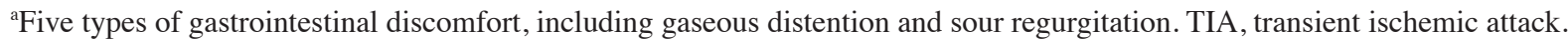

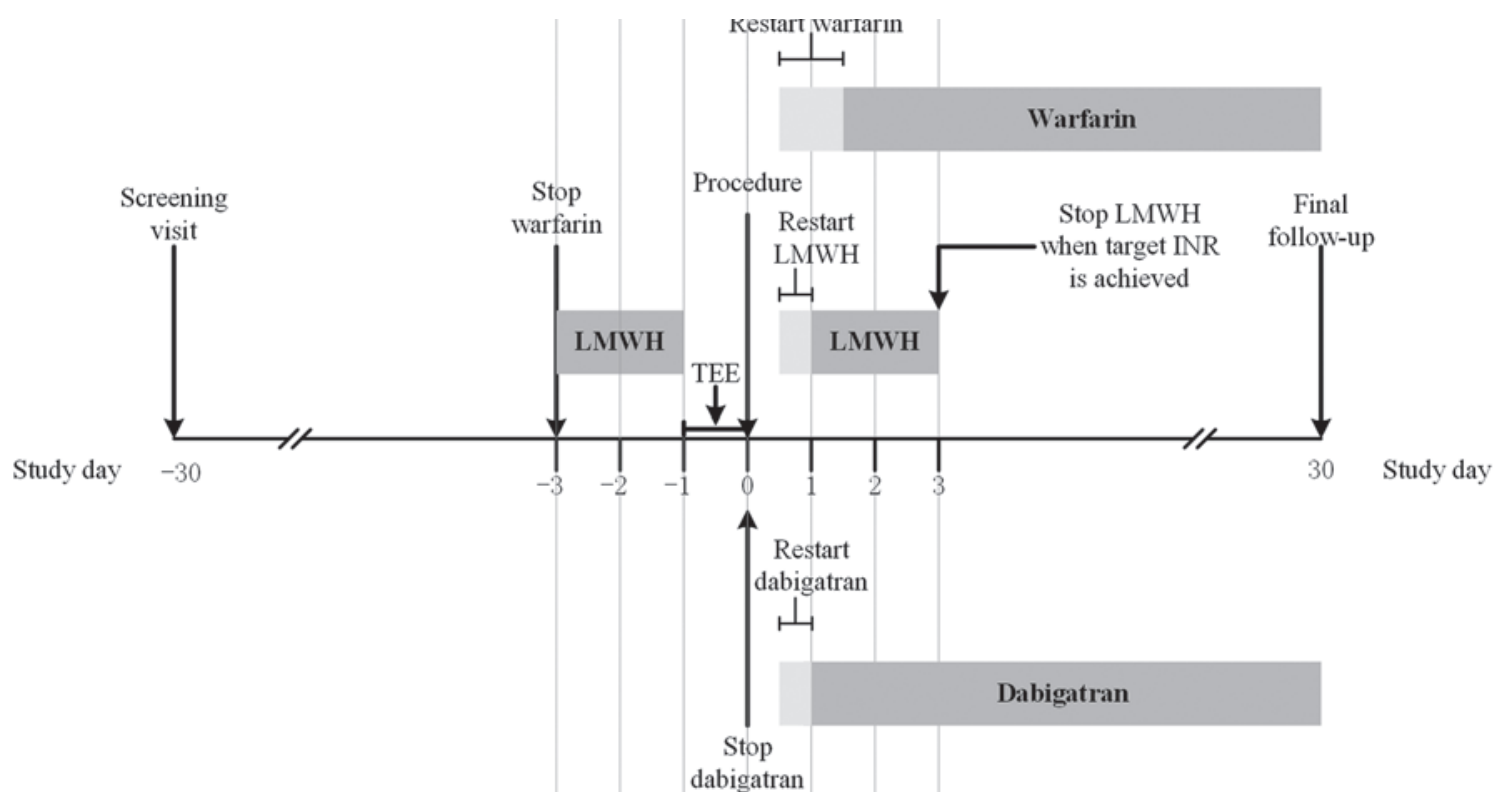

Figure 1. Study design flow chart. Screening visits occurred between 30 days and 3 days prior to the ablation procedure. For all patients, the absence of intra-cardiac thrombus was proved by TEE prior to the procedure. In group W, warfarin treatment was discontinued and the administration of the LMWH was initiated 3 days prior to the procedure. LMWH was restarted on the evening of the procedure and continued until an INR of 2.0-3.0 was achieved. Warfarin treatment was restarted on the evening of or the day after the procedure. In group D, dabigatran was not discontinued until the morning of the procedure and was restarted $4 \mathrm{~h}$ following hemostasis. The final patient follow-up occurred 30 days following the ablation procedure. TEE, transesophageal echocardiography; LMWH, low-molecular-weight heparin.

no patients in the warfarin group. No major bleeding complications were observed in any patients within the study population. The incidence of minor bleeding complications was 23 in group D (17\%) and 11 in group W (11\%, P>0.05). In addition, 11 patients receiving dabigatran treatment developed a groin hematoma compared with five patients receiving warfarin. All of these patients underwent an ultrasound scan that excluded the presence of a pseudo-aneurysm or an arteriovenous $(\mathrm{AV})$ fistula. As a result, one patient in each of the groups was indicated to have an AV fistula. A hemothorax was observed in one patient in the dabigatran group and one patient from the warfarin group. Hemoptysis occurred in one patient who received dabigatran treatment, and the patient developed a large groin hematoma two days following the procedure and a high fever of $38.6^{\circ} \mathrm{C}$. Dabigatran was discontinued imminently. Two days later, massive hemoptysis occurred and pulmonary computed tomography (CT) presented bilateral pulmonary frosted glass. At three days later, no hemoptysis occurred and a lung CT presented markedly reduced frosted glass. Overall, there were no significant 
differences in complication rates between the two groups (P>0.05; Table II).

\section{Discussion}

Stroke is the most serious complication in patients with AF. Therefore, in AF patients with a CHADS2 score $\geq 2$, conventional OAC is recommended to reduce the risk of stroke $(13,14)$. During the period of anticoagulation therapy, the risk of both stroke and bleeding complications are highest when OAC is initiated. During the initiation of $\mathrm{OAC}$, there is a theoretical transient hypercoagulable state, because the vitamin $\mathrm{K}$-dependent anticoagulant proteins $\mathrm{C}$ and $\mathrm{S}$ are decreased, while the vitamin $\mathrm{K}$-dependent procoagulant factors II and X remain elevated due to their longer half-lives (15). Bridging anticoagulation therapy is designed to minimize the risk of thromboembolism in high-risk patients when anticoagulation therapy is suspended and to minimize the risk of bleeding following procedures, and it is usually used in patients receiving warfarin treatment when warfarin has been discontinued and the INR falls below the therapeutic range (16). During the interruption of warfarin treatment, typically LMWH bridging anticoagulation therapy can be given to minimize the interval that patients do not in a state of anticoagulation, with the purpose of decreasing the risk of perioperative stroke $(8,16)$. In patients who require temporary interruption of warfarin therapy for invasive procedures, a standardized periprocedural bridging anticoagulant therapy with subcutaneous LMWH is feasible and associated with a low risk of thromboembolic and major bleeding complications $(17,18)$. Some studies have assessed perioperative bridging with LMWH, however, the practice guidelines have provided weak and inconsistent recommendations regarding whether bridging anticoagulation is necessary during perioperative warfarin interruption (19-21). Previous research has indicated that use of bridging anticoagulation is associated with an increased risk of bleeding and adverse events after interruption $(22,23)$ and in patients who interrupted dabigatran or warfarin in the RE-LY trial, bridging anticoagulation appeared to increase the risk for major bleeding irrespective of dabigatran or warfarin interruption (24). The use of interrupted warfarin bridging with LMWH has been widely used, although studies have suggested that continuous warfarin treatment protects against the risk of periprocedural stroke with no increased bleeding risk $(8,22-24)$.

Given the growing concern regarding hemorrhagic complications associated with bridging therapies, some experts suggest that bridging should only be considered in those at highest risk for thrombosis (11). A recent study indicated that performing AF catheter ablation with uninterrupted warfarin in patients at high risk for stroke and with nonparoxysmal AF reduced the periprocedural stroke and bleeding complications compared to bridging therapy (7). The results of the present study suggested that interrupted warfarin bridged with LMWH is safe and effective among the low-risk population compared to anticoagulation with dabigatran. In the current study, there was no difference in thromboembolic events in the two anticoagulant groups. The incidence and outcomes of bleeding complications were also similar between the two groups. Of note, some of the complications were likely the result of technical or mechanical complications, as opposed to the choice of anticoagulant.

In the present study, the mean CHADS2 score was $0.68 \pm 0.80$ vs. $0.77 \pm 0.89$ in dabigatran and warfarin groups, respectively $(\mathrm{P}>0.05)$. There were 17 patients with $\mathrm{CHADS} 2$ $\geq 2(12 \%)$ in the dabigatran group and 19 patients in the warfarin group $(19 \%, \mathrm{P}>0.05)$. This also applied to the CHA2DS2VASc score and HAS-BLED score. As such, the entire population tended to have a low risk of both thromboembolic and bleeding events. Anticoagulation-experienced is defined as the total lifetime use of the anticoagulant for more than two months. There was no difference between the two groups in ratio of the anticoagulation-experienced $(\mathrm{P}>0.05)$ and only $>20 \%$ of the patients were treated with OAC at baseline. Asian patients are more sensitive to the anticoagulant effects of warfarin and have higher rates of bleeding when they are in the therapeutic range (12). Therefore, frequent therapeutic monitoring and concern about bleeding events remains an important barrier to the appropriate use of anticoagulant therapy in the Chinese population $(25,26)$. The cost of routine dabigatran or rivaroxaban anticoagulation is too high to be affordable for ordinary patients. Therefore, it is convenient and likely safe to leave low-risk patients on aspirin or no OAC pre-ablation. In addition, the interrupted anticoagulation strategy with LMWH bridging was safe and effective for the AF patients who had a low risk score.

Despite a recent trend towards uninterrupted $\mathrm{OAC}$ for $\mathrm{AF}$ ablation $(7,27,28)$, interrupted OAC provides several advantages. Some patients on uninterrupted warfarin will not have a stable INR pre-ablation and may arrive with a subtherapeutic or supratherapeutic INR (29). With the NOACs, there is no test to verify patient compliance pre-ablation, and the lack of proven reversal agents may add additional risk if they are not interrupted. In addition, bleeding complications are easier to handle with interrupted OAC. The use of uninterrupted warfarin was followed by an era of using interrupted warfarin. Thus, published retrospective comparisons have been conducted by examining these two different time periods of AF ablation. It was demonstrated that some of the apparent advantages of uninterrupted warfarin may instead be due to improvements in ablation safety over time. Arshad et al (30) recently compared peri-ablation anticoagulation at four experienced AF ablation centers and indicated that major complications (stroke/TIA, pericardial tamponade, major bleeding and surgical intervention) occur more frequently in the uninterrupted warfarin group (4.3\%) vs. both the dabigatran group (0.8\%) and the bridged warfarin group $(2.6 \%)(\mathrm{P}<0.01)$.

In conclusion, the administration of dabigatran during AF ablation procedures did not cause any significantly different effects on the safety and efficiency as compared to those of the conventional anticoagulation with warfarin bridged with LMWH for patients with a low CHADS2 score. Low-risk patients may remain on aspirin or no OAC pre-ablation and do not need to receive warfarin or dabigatran before ablation.

\section{Acknowledgements}

The present study was supported by the National Natural Science Foundation of China (grant no. 81270238) and the 
Scientific Research Foundation for the Doctoral Degree, State Education Ministry of China (grant no. 20130131110065).

\section{References}

1. Ball J, Carrington MJ, McMurray JJ and Stewart S: Atrial fibrillation: Profile and burden of an evolving epidemic in the $21 \mathrm{st}$ century. Int J Cardiol 167: 1807-1824, 2013

2. Alonso A and Bengtson LG: A rising tide: The global epidemic of atrial fibrillation. Circulation 129: 829-830, 2014.

3. Cappato R, Calkins H, Chen SA, Davies W, Iesaka Y, Kalman J, Kim YH, Klein G, Natale A, Packer D, et al: Updated worldwide survey on the methods, efficacy, and safety of catheter ablation for human atrial fibrillation. Circ Arrhythm Electrophysiol 3: $32-38,2010$

4. Morillo CA, Verma A, Connolly SJ, Kuck KH, Nair GM, Champagne J, Sterns LD, Beresh H, Healey JS and Natale A; RAAFT-2 Investigators: Radiofrequency ablation vs antiarrhythmic drugs as first-line treatment of paroxysmal atrial fibrillation (RAAFT-2): A randomized trial. JAMA 311: 692-700, 2014.

5. Gaita F, Caponi D, Pianelli M, Scaglione M, Toso E, Cesarani F, Boffano C, Gandini G, Valentini MC, De Ponti R, et al: Radiofrequency catheter ablation of atrial fibrillation: A cause of silent thromboembolism? Magnetic resonance imaging assessment of cerebral thromboembolism in patients undergoing ablation of atrial fibrillation. Circulation 122: 1667-1673, 2010.

6. Phan K, Wang N, Pison L, Kumar N, Hitos K and Thomas SP: Meta-analysis of dabigatran vs warfarin in patients undergoing catheter ablation for atrial fibrillation. Int J Cardiol 189: 199-203, 2015.

7. Di Biase L, Burkhardt JD, Santangeli P, Mohanty P, Sanchez JE, Horton R, Gallinghouse GJ, Themistoclakis S, Rossillo A, Lakkireddy D, et al: Periprocedural stroke and bleeding complications in patients undergoing catheter ablation of atrial fibrillation with different anticoagulation management: Results from the role of coumadin in preventing thromboembolism in Atrial Fibrillation (AF) patients undergoing catheter ablation (COMPARE) randomized trial. Circulation 129: 2638-2644, 2014.

8. Douketis JD, Spyropoulos AC, Kaatz S, Becker RC, Caprini JA, Dunn AS, Garcia DA, Jacobson A, Jaffer AK, Kong DF, et al: Perioperative bridging anticoagulation in patients with atrial fibrillation. N Engl J Med 373: 823-833, 2015.

9. You JJ, Singer DE, Howard PA, Lane DA, Eckman MH, Fang MC, Hylek EM, Schulman S, Go AS, Hughes M, et al: Antithrombotic therapy for atrial fibrillation: antithrombotic therapy and prevention of thrombosis, 9th ed: American college of chest physicians evidence-based clinical practice guidelines. Chest 141 (Suppl 2): e531S-e575S, 2012.

10. van Veen JJ and Makris M: Management of peri-operative anti-thrombotic therapy. Anaesthesia 70 (Suppl 1): S58-S67, 2015.

11. Kim TH, Kim JY, Mun HS, Lee HY, Roh YH, Uhm JS, Pak HN Lee $\mathrm{MH}$ and Joung B: Heparin bridging in warfarin anticoagulation therapy initiation could increase bleeding in non-valvular atrial fibrillation patients: A multicenter propensity-matched analysis. J Thromb Haemost 13: 182-190, 2015.

12. Hori M, Connolly SJ, Zhu J, Liu LS, Lau CP, Pais P, Xavier D, Kim SS, Omar R, Dans AL, et al: Dabigatran versus warfarin: Effects on ischemic and hemorrhagic strokes and bleeding in Asians and non-Asians with atrial fibrillation. Stroke 44 1891-1896, 2013.

13. Crandall MA, Bradley DJ, Packer DL and Asirvatham SJ: Contemporary management of atrial fibrillation: Update on anticoagulation and invasive management strategies. Mayo Clin Proc 84: 643-662, 2009.

14. Gage BF, Waterman AD, Shannon W, Boechler M, Rich MW and Radford MJ: Validation of clinical classification schemes for predicting stroke: Results from the National Registry of Atrial Fibrillation. JAMA 285:2864-2870, 2001.

15. Zeuthen EL, Lassen JF and Husted SE: Is there a hypercoagulable phase during initiation of antithrombotic therapy with oral anticoagulants in patients with atrial fibrillation? Thromb Res 109: 241-246, 2003.
16. Baron TH, Kamath PS and McBane RD: Management of antithrombotic therapy in patients undergoing invasive procedures. N Engl J Med 368: 2113-2124, 2013.

17. Douketis JD, Johnson JA and Turpie AG: Low-molecular-weight heparin as bridging anticoagulation during interruption of warfarin: Assessment of a standardized periprocedural anticoagulation regimen. Arch Intern Med 164: 1319-1326, 2004.

18. Kovacs MJ, Kearon C, Rodger M, Anderson DR, Turpie AG, Bates SM, Desjardins L, Douketis J, Kahn SR, Solymoss S and Wells PS: Single-arm study of bridging therapy with low-molecular-weight heparin for patients at risk of arterial embolism who require temporary interruption of warfarin. Circulation 110: 1658-1663, 2004.

19. BRIDGE Study Investigators: Bridging anticoagulation: Is it needed when warfarin is interrupted around the time of a surgery or procedure? Circulation 125: e496-e498, 2012.

20. Patel JP and Arya R: The current status of bridging anticoagulation. Br J Haematol 164: 619-629, 2014.

21. Douketis JD, Spyropoulos AC, Spencer FA, Mayr M, Jaffer AK, Eckman MH, Dunn AS and Kunz R; American College of Chest Physicians: Perioperative management of antithrombotic therapy: Antithrombotic Therapy and Prevention of Thrombosis, 9th ed: American College of Chest Physicians Evidence-Based Clinical Practice Guidelines. Chest 141 (2 Suppl): e326S-e350S 2012.

22. Steinberg BA, Peterson ED, Kim S, Thomas L, Gersh BJ, Fonarow GC, Kowey PR, Mahaffey KW, Sherwood MW, Chang $\mathrm{P}$, et al: Use and outcomes associated with bridging during anticoagulation interruptions in patients with atrial fibrillation: Findings from the Outcomes Registry for Better Informed Treatment of Atrial Fibrillation (ORBIT-AF). Circulation 131: 488-494, 2015.

23. Siegal D, Yudin J, Kaatz S, Douketis JD, Lim W and Spyropoulos AC: Periprocedural heparin bridging in patients receiving vitamin $\mathrm{K}$ antagonists: Systematic review and meta-analysis of bleeding and thromboembolic rates. Circulation 126: 1630-1639, 2012.

24. Douketis JD, Healey JS, Brueckmann M, Eikelboom JW, Ezekowitz MD, Fraessdorf M, Noack H, Oldgren J, Reilly P, Spyropoulos AC, et al: Perioperative bridging anticoagulation during dabigatran or warfarin interruption among patients who had an elective surgery or procedure. Substudy of the RE-LY trial. Thromb Haemost 113: 625-632, 2015.

25. Sabir I, Khavandi K, Brownrigg J and Camm AJ: Oral anticoagulants for Asian patients with atrial fibrillation. Nat Rev Cardiol 11: 290-303, 2014.

26. Eikelboom JW and Hart RG: Intensity and quality of warfarin anticoagulation in chinese patients: Setting the record straight. Stroke 46: 5-6, 2015.

27. Hussein AA, Martin DO, Saliba W, Patel D, Karim S, Batal O, Banna M, Williams-Andrews M, Sherman M, Kanj M, et al: Radiofrequency ablation of atrial fibrillation under therapeutic international normalized ratio: A safe and efficacious periprocedural anticoagulation strategy. Heart Rhythm 6: 1425-1429, 2009.

28. Wazni OM, Heheiry S, Fahmy T, Barrett C, Hoa S, Patel D, Di Biase L, Martin DO, Kanj M, Arruda M, et al: Atrial fibrillation ablation in patients with therapeutic international normalized ratio: Comparison of strategies of anticoagulation management in the periprocedural period. Circulation 116: 2531-2534, 2007.

29. Nam D, Sadhu A, Hirsh J, Keeney JA, Nunley RM and Barrack RL: The use of warfarin for DVT prophylaxis following hip and knee arthroplasty: How often are patients within their target INR range? J Arthroplasty 30: 315-319, 2015.

30. Arshad A, Johnson CK, Mittal S, Buch E, Hamam I, Tran T, Shaw RE, Musat D, Preminger M, Sichrovsky T, et al: Comparative safety of periablation anticoagulation strategies for atrial fibrillation: Data from a large multicenter study. Pacing Clin Electrophysiol 37: 665-673, 2014. 